\title{
openheart Time-based measures of treatment effect: reassessment of ticagrelor and clopidogrel from the PLATO trial
}

\author{
Andrea Bellavia, ${ }^{1}$ Lars Wallentin, ${ }^{2,3}$ Nicola Orsini, ${ }^{4}$ Stefan K James, ${ }^{2,3}$ \\ Christopher P Cannon, ${ }^{5,6}$ Anders Himmelmann, ${ }^{7}$ Johan Sundström, ${ }^{2,3}$ \\ Henrik Renlund, ${ }^{3}$ Per Lytsy ${ }^{8}$
}

To cite: Bellavia A,

Wallentin L, Orsini N, et al. Timebased measures of treatment effect: reassessment of ticagrelor and clopidogrel from the PLATO trial. Open Heart 2017;4:e000557. doi:10.1136/ openhrt-2016-000557

Received 3 November 2016 Revised 18 January 2017 Accepted 14 February 2017

\section{(a) CrossMark}

${ }^{1}$ Institute of Environmental Medicine, Karolinska Institutet, Stockholm, Sweden

${ }^{2}$ Department of Medical Sciences, Cardiology, Uppsala University, Uppsala, Sweden ${ }^{3}$ Uppsala Clinical Research Center, Uppsala University, Uppsala, Sweden

${ }^{4}$ Department of Public Health Science, Karolinska Institutet, Stockholm, Sweden

${ }^{5}$ Division of Cardiovascular, Brigham and Women's Hospital, Boston, Massachusetts, USA

${ }^{6}$ HarvardClinical Research Institute, Boston, Massachusetts USA

${ }^{7}$ AstraZeneca Research and Development, Gothenburg, Sweden

${ }^{8}$ Department of Public Health and Caring Sciences, Uppsala University, Uppsala, Sweden

Correspondence to Dr Andrea Bellavia; abellavi@ hsph.harvard.edu

\section{ABSTRACT}

Objective Treatment effects to binary endpoints using time-to-event data in randomised controlled trials are typically summarised by reporting HRs derived with Cox proportional hazard models. Alternative and complementary methods include summarising the between-treatment differences on the metric time scale, quantifying the effect as delay of the event (DoE). The aim of this study was to reassess data from the PLATO study expressing the effects as the time by which the main outcomes are delayed or hastened due to treatment. Methods PLATO was a randomised controlled doubleblind multicentre study ( $n=18,624)$, conducted between 2006 and 2008, which demonstrated superiority of the antiplatelet treatment ticagrelor over clopidogrel in reducing risk of several cardiovascular events. In the present study, four of the main PLATO outcomes were reassessed by calculating the time by which an event may be delayed due to the treatment.

Results The effects of ticagrelor, as compared with clopidogrel, consisted of a substantial delay of the evaluated outcomes, ranging from 83 to 98 days over 400-day follow-up. The Delay of Events Curves showed that the effects progressively increased over time, and the significant findings were concordant with those presented in the original PLATO study.

Conclusions This study confirmed evidence of a beneficial effect of ticagrelor over clopidogrel, and provided the magnitude of such effects in terms of delayed event time. Investigating time-to-event data with a percentile approach allows presenting treatment effects from randomised controlled studies as absolute measures of the time by which an event may be delayed due to the treatment.

Trial registration number PLATO (www.clinicaltrials.gov; NCT00391872); Results.

\section{INTRODUCTION}

Patients with acute coronary syndrome (ACS), with or without ST-segment elevation, benefit from antiplatelet therapy, which reduces the risk of major cardiovascular adverse events and premature cardiovascular death. In 2009, the PLATO study demonstrated that ticagrelor, a new reversibly binding and direct-acting oral antagonist of the ADP receptor, significantly

\section{KEY MESSAGES}

What is already known about this subject? PLATO was a randomised controlled double-blind multicentre study which demonstrated superiority of the antiplatelet treatment ticagrelor over clopidogrel in reducing risk of several cardiovascular events.

What does this study add?

Four of the main PLATO outcomes were reassessed by calculating the time by which an event may be delayed due to the treatment. Ticagrelor provides substantial benefits by delaying the events in the range of $80-100$ days.

How might this impact on clinical practice? Findings from the current study may add substantial information to the pharmaco-pathophysiological understanding of how the treatment provides its effect and how that relates to time on treatment.

reduces the risk of the composite of death from vascular causes, myocardial infarction (MI) or stroke as well as the risk of vascular death, compared with clopidogrel in patients with ACS. ${ }^{1}$ Ticagrelor has since then been included as an antiplatelet treatment option in the treatment guidelines. ${ }^{2-4}$

The PLATO study was a state-of-the art randomised controlled multicentre study that investigated several binary outcomes comparing a new treatment alternative to the best available treatment clopidogrel. The effect of ticagrelor over clopidogrel was assessed using Cox proportional hazard models, which is the typical statistical approach when investigating binary endpoints using time-to-event data. Results from such analyses, in agreement with the CONSORT recommendations, ${ }^{5}$ are conventionally presented by displaying the survival curves derived with the Kaplan-Meier method, and by summarising the effect measures in terms of absolute event rates as well as HRs and 95\% CIs. For example, in the 
original PLATO study, it was reported that the primary endpoint of overall mortality occurred in $4.5 \%$ of study subjects in the ticagrelor group, and in 5.9\% in the clopidogrel group over the follow-up period. The comparison between the groups was presented by reporting an HR of 0.78 (95\% CI 0.69 to 0.89 ).

Despite the well-established advantages of estimating HRs with Cox regression, the solitary use of HRs to present treatment effects does not come without some limitations, which have been summarised in details. ${ }^{6-10}$ Several authors have underlined the importance of complementing regular statistical analyses with other effect measures, possibly providing the time dimension of the association of interest. ${ }^{6-8}$ One possibility is to evaluate the percentiles of the survival function presenting results in terms of survival percentile differences. ${ }^{8-10}$ Such measures of association can be obtained from the KaplanMeier curve and express the delay in time by which a specific proportion of cases is achieved by different treatment groups. ${ }^{10}$ One of the main advantages of such measure is that any percentile can be estimated, which is extremely useful in situations where the fraction of events is low or the follow-up time is short. ${ }^{8}$ The development of multivariable statistical methods for survival percentiles, such as Laplace regression, ${ }^{11}$ has extended the potentials of investigations in which multivariable adjustment is required or interactions need to be assessed. ${ }^{9}$ ${ }^{12}$ Presenting the effect of a covariate or treatment as a gain (or loss) in disease-free time is further believed to be an intuitive measure, easy to interpret for laymen. Such a measure could, thus, be of value in clinical decision making when patients are to decide to initiate and adhere to a proposed treatment. ${ }^{13} 14$

The overall aim of the present study is to investigate the effects of ticagrelor in the PLATO study in terms of delay of the event as compared with clopidogrel. The study also has the aim to illustrate the advantages and clinical relevance of a complementary estimation of utility method to quantify treatment effects in terms of delayed event time.

\section{METHODS}

PLATO (www.clinicaltrials.gov; NCT00391872; currently in Results stage) was a randomised controlled doubleblind multicentre study including 18624 patients from 862 centres in 43 countries during 2006-2008. Details about the design ${ }^{15}$ and the main results ${ }^{1}$ have been published previously. This study uses the same study sample as the original study, which included patients hospitalised for ACS, with or without ST-segment elevation, with an onset within the preceding 24 hours. Patients were randomly assigned to receive ticagrelor, $90 \mathrm{mg}$ twice daily ( $\mathrm{n}=9333$ ), or clopidogrel $75 \mathrm{mg}$ daily $(\mathrm{n}=9291)$ and were followed for a 12-month study period.

Appropriate national and institutional regulatory authorities and ethics committees approved the study design of the original study and all participants provided written informed consent. The present study was approved by the PLATO Publications Committee.

\section{The main outcomes}

The following reported outcomes in PLATO are re-evaluated in the present study: (1) time to first occurrence of the composite of death from vascular causes, MI (excluding silent MI) or stroke; (2) time to first occurrence of MI (including silent MI); (3) cardiovascular death/MI (including silent MI); (4) all-cause mortality/ MI and stroke; (5) total major bleeding, as defined in the original PLATO study; and (6) non-CABG (coronary-artery bypass grafting) major bleeding events.

\section{Statistical analysis}

The effect of ticagrelor over clopidogrel was assessed in terms of Delay of Events (DoE), differences in survival time by which specific proportions of cases are achieved by the two treatment groups. ${ }^{10}$ DoE was estimated by modelling survival percentiles with Laplace regression, a statistical method for censored quantiles. ${ }^{9}$ For all the six investigated outcomes, the cumulative incidence curves for the two treatment groups were estimated by taking the complement of the survival curves calculated with the Kaplan-Meier method. From the cumulative incidence, it is possible to assess the highest percentile that can be estimated, that is, the achieved fraction of cases in the superior treatment. Estimation of survival percentiles beyond the highest observed percentile would require data extrapolation. Given a specific percentile corresponding to a time point when an event would occur taking the lesser efficient treatment, that is, clopidogrel, the DoE expresses the benefit in terms of how long the event is delayed due to the superior treatment (ie, ticagrelor), that is, the increase in event-free time. When inevitable health outcomes, such as mortality for all causes, are investigated, the DoE depicts a prolonged survival time. A negative DoE would imply harm, that is, that the event occurs earlier than in the compared group.

For all six scenarios, DoE and corresponding 95\% CIs were then estimated by simultaneously fitting unadjusted Laplace regression on all the observed percentiles. The treatment effect as measured by the survival percentile difference was then plotted versus the survival percentile in the control group. In our case, results were graphically reported by plotting the delay in survival time in the ticagrelor group versus survival time in the clopidogrel group. The percentile approach directly connects delay times and percentiles, so that it is also possible to think of the delay as a function of treatment time. The graphical presentation that we provide depicts this relationship by presenting DoEs as a function of survival time in the group that has the lowest cumulative incidence at the end of the treatment period.

All statistical analyses were performed with the laplace function in $\mathrm{R}$, currently incorporated in the ctqr package. ${ }^{16}$ (The laplace function is still available upon request at http://www.imm.ki.se/biostatistics/laplace/). 


\begin{tabular}{|c|c|c|}
\hline Variable & Ticagrelor $n=9333$ & Clopidogrel $n=9291$ \\
\hline $\begin{array}{l}\text { Age, years (median, } \\
\text { IQR) }\end{array}$ & $62.0(54.0-71.0)$ & $62.0(54.0-71.0)$ \\
\hline Age $>75$ years & $1396(15.0 \%)$ & $1482(16.0 \%)$ \\
\hline Female & $2655(28.4 \%)$ & $2633(28.3 \%)$ \\
\hline Weight (median, IQR) & $80.0(70.0-90.0)$ & $80.0(70.0-90.0)$ \\
\hline Weight $<60 \mathrm{~kg}$ & $652(7.0 \%)$ & $660(7.1 \%)$ \\
\hline BMI (median, IQR) & $27.4(24.7-30.5)$ & $27.3(24.7-30.4)$ \\
\hline \multicolumn{3}{|l|}{ Race } \\
\hline White & $8566(91.8 \%)$ & $8511(91.6 \%)$ \\
\hline Black & $115(1.2 \%)$ & $114(1.2 \%)$ \\
\hline Asian & $542(5.8 \%)$ & $554(6.0 \%)$ \\
\hline Other & $109(1.2 \%)$ & $112(1.2 \%)$ \\
\hline Current smoker & $2326(24.9 \%)$ & $3318(35.7 \%)$ \\
\hline Diabetes prevalence & $2326(24.9 \%)$ & $2336(25.1 \%)$ \\
\hline
\end{tabular}

BMI, body mass index.

\section{RESULTS}

The study population is the same as in the original PLATO study where baseline characteristics were balanced in both treatment arms (table 1). For a more detailed description of baseline characteristics, including presence of cardiovascular risk factors, comorbidity and diagnostic findings, the reader can refer to the original publication. ${ }^{1}$

Figure 1 presents the cumulative incidence curves and DoE graphs for the six investigated outcomes (panels A-F), using the clopidogrel group as reference. To clarify the meaning and interpretation of the DoE, results on the first outcome are further represented in details in Figure 2. When focusing on time to the composite of death from vascular causes, MI (excluding silent MI) or stroke (figures 1A, 2) the delay of event due to ticagrelor as compared with clopidogrel at the fourth, sixth and eighth percentiles is estimated at 4 (95\% CI 0 to 9 ), 30 (95\% CI 7 to 53$)$ and 73 (95\% CI 20 to 127$)$ days. At the end of 1-year treatment, when the clopidogrel group is at the 10th percentile, the delay of event was 83 days (10th DoE $=83$ days; $95 \%$ CI 37 to 128$)$. The Delay of eEvents at the 10th percentile is illustrated by a horizontal line in the Kaplan-Meier graph. Within the clopidogrel group, $10 \%$ of the study subjects experienced outcome 1 within 223 days, and the same fraction of cases was observed 83 days later among subjects treated with ticagrelor. MI was observed in $5 \%$ of the patients, and the delay of the event was up to 84 days at the fifth percentile (fifth $\mathrm{DoE}=84$ days; $95 \%$ CI 31 to 136) (figure 1B). Death from vascular causes, MI and stroke was observed in $9 \%$ of the study population, and the delay in the event associated with the ticagrelor treatment was up to almost 100 days (ninth DoE $=98$ days; $95 \%$ CI 50 to 146) (figure 1C). Similar proportions and delay in survival were observed for the composite endpoint of all-cause mortality, MI and stroke (ninth DoE=94 days; 95\% CI 49 to 139) (figure 1D). Total bleeding and non-CABG-related major bleedings were the only outcomes in which ticagrelor did not show beneficial effects (figure 1E,F). The negative Delay of eEvents, consistently observed over follow-up, was not statistically significant for total bleeding (10th DoE=-29 days; $95 \% \mathrm{CI}$ 88 to 30 ) but was statistically significant for non-CABG-related bleeding (fifth DoE=-95 days; $95 \%$ CI 170 to 30 ).

\section{DISCUSSION}

The main goal of this study was to re-evaluate data from the high-quality PLATO clinical trial to quantify, in terms of delayed survival, the beneficial effects of ticagrelor treatment over clopidogrel. By evaluating the percentiles of survival, we quantified the established information of relative efficacy of the treatment in absolute terms of delayed time to the adverse events. We observed that ticagrelor provides substantial benefits by delaying the events in the range of 80-100 days. Our results support the hypothesis that additional measures to quantify the treatment/outcome effect in terms of time improve the understanding of the treatment effects over follow-up.

When evaluating time-to-event outcome, the common way of summarising results, such as estimating HRs with Cox proportional hazard regression, may be subject to some limitations. First, the treatment effect is commonly reported as a single $\mathrm{HR}$, implicitly assuming a fixed effect over the follow-up. However, since the HR may change over time, information may be lost and results become dependent on the time point of the analysis. ${ }^{6}$ The variation of a treatment's effect may be of special concern in studies where the occurrence of the outcome is non-linear over time, such as the case of postsurgery and postinfarction studies, where there typically is a high initial risk of death/adverse events followed by a gradual decrease/stabilisation over time. Methods to incorporate time-varying effects in a Cox model, thus presenting HRs as a function of time, have been developed, but these are rarely used in regular practice, and presenting results with a single HR solidly remains the most common way to summarise the between-group difference in time-to-event analysis. Moreover, the HR is a relative effect measure, and despite its methodological justification a solitary HR is not sufficient to support clinical decisions, as it is uninformative of the absolute risk. Studies have shown that relative measures of effect, when presented to patients and doctors, exaggerate understanding of the effect compared with absolute measures. ${ }^{17-19}$

Over the possible additional measures presented in the literature, the evaluation of survival percentiles provides various advantages that were documented in this study. First, it is suitable in studies with low-risk events, as researchers may focus the analysis at low percentiles without any data extrapolation. Despite being a high-risk patient group, the incidence rates in the PLATO trial were rather low for the reported outcomes. In this study, by focusing on survival percentiles, we could evaluate 

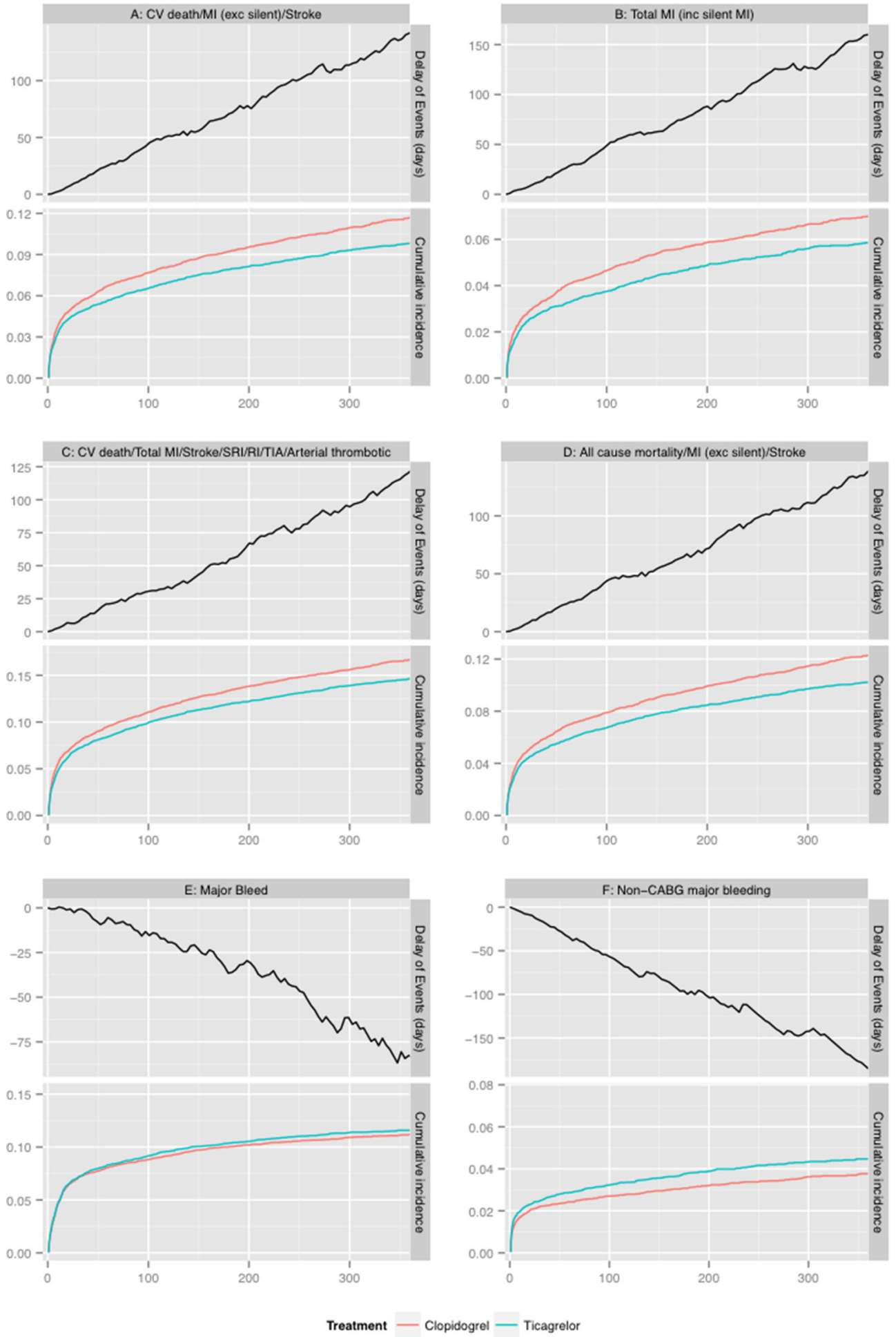

Figure 1 Cumulative incidence in clopidogrel and ticagrelor groups, and Delay of eEvents associated with ticagrelor use for death from vascular causes/MI or stroke (A), $\mathrm{MI}(\mathrm{B})$, death from vascular causes, $\mathrm{MI}$, stroke (C), death from any cause, $\mathrm{MI}$ or stroke (D), total major bleeding (E) and non-CABG major bleeding (F). CABG, coronary-artery bypass grafting; MI, myocardial infarction; TIA, transient ischaemic attack; CV, cardiovascular; SRI, serious recurrent ischaemia; RI, recurrent ischaemia.

differences in survival, for each outcome, only relying on the observed fraction of cases. Another important quality of the percentile approach is that it gives an estimate of the magnitude of the effect, quantified in terms of time. For instance, we observed maximum delays of the event up to 83-98 days. These effects are substantial, given that the follow-up-time was 360 days, and provide evidence of the treatment benefits in a way that is not captured by classical approaches. The DoE depicts the effect in an absolute matter, which may be more comprehensible for patients and laymen and, thus, of potential value in clinical decision making. Appealing features of the percentile 


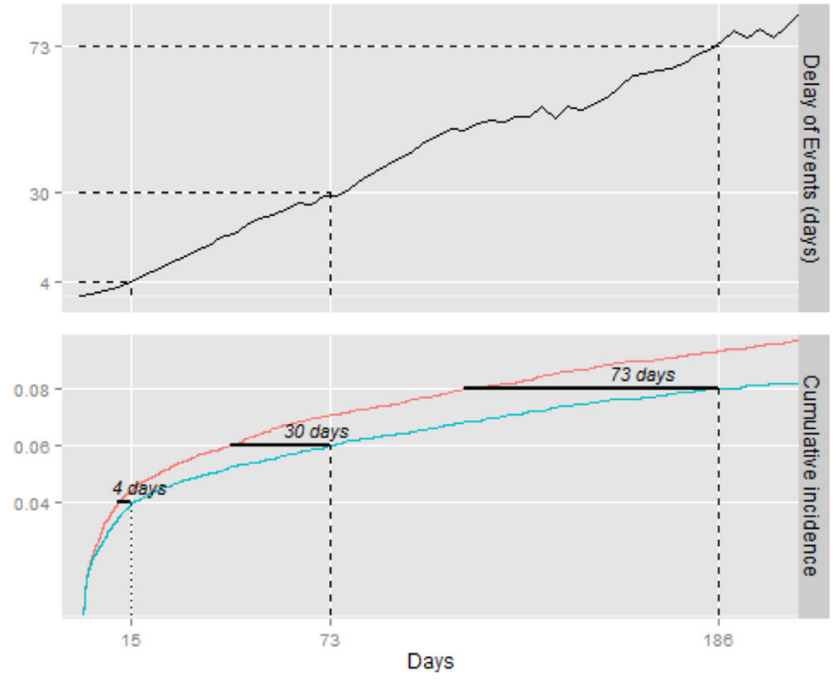

Treatment - Clopidogrel - Ticagrelor

Figure 2 Cumulative incidence in clopidogrel and ticagrelor groups, and Delay of eEvents associated with ticagrelor use for death from vascular causes/myocardial infarction or stroke.

approach also include the simple interpretation of the graphical display, which complements the well-known Kaplan-Meier estimator. The curves in figure 1A-D intuitively display the beneficial effects of ticagrelor over clopidogrel, effects that are consistent during the entire follow-up. Such information could not be easily obtained and conveyed when presenting the treatment effect using a single HR.

Recent developments in the statistical literature for censored quantile regression allow estimating survival percentiles in a regression-based framework. ${ }^{11}$ Among the possible methods, the use of Laplace regression, where coefficients obtained from the model are directly interpreted in terms of Delay of eEvents associated with the predictor of interest, provides important features. Laplace directly focuses on the survival function by estimating the percentiles of interest without requiring any specific shape for the overall survival curve. ${ }^{11}$ The model uses a distributional assumption on the errors (asymmetric Laplace distribution). Nevertheless, the model has shown good performances under different scenarios. ${ }^{20-22} \mathrm{~A}$ robust estimation via a gradient search maximisation algorithm is used for its estimation. ${ }^{21}$ Laplace provides all modelling advantages such as multivariable adjustment ${ }^{9}$ or interaction assessment, ${ }^{12}$ can be used with different time scales. ${ }^{20}$ Methods for censored quantiles based on the Laplace distribution are available as a user-friendly command in the Stata statistical software (up to version 14), ${ }^{22}$ and in the $c t q r$ package in $\mathrm{R}$, which can also incorporate truncated data. ${ }^{16}$ Other conditional censored quantile methods can be estimated in SAS with PROC QUANTLIFE (up to version 9.3), and in $\mathrm{R}$ with the function $\mathrm{crq}$ within the package quantreg. The performances and advantages of the methods have been presented, in recent years, by different application on both real $^{23-25}$ and simulated data. ${ }^{20-22}$ While possibly all percentiles can be estimated, one should avoid estimating percentiles beyond the observed range that would require a consistent amount of data extrapolation. Future methodological research should extend the method and evaluate its performances in relevant settings such as those situations where competing events are taken into account

By definition, the percentile approach reports associations at specific percentiles. The individual interpretation of a DoE graph is therefore conditional on having experienced the event at specific time point. The DoE curve, therefore, only applies to patients who could have been included in the study and who would have developed the event sometime during the follow-up. More generally, this is the target group for a preventive treatment-only patients who would have an event when untreated may benefit from treatment. In shared clinical decision making, reporting results in terms of Delay of eEvents would allow communicating to the patients: 'You have an increased risk of having (the event) in the next (time period). This curve gives you an estimate of how much the event may be delayed if you take the treatment for (time period).' The potential use of the DoE in shared clinical decision making, nevertheless, remains to be further investigated.

This study also had the analytical purpose of comparing if the statistical findings from the original PLATO study would be concordant with statistical findings for the same outcomes using the percentile approach. As expected, findings from this study, while providing an absolute measure of the magnitude of the effect, were consistent with the original study results. As illustrated in table 2, the significant findings of the effect as HRs in the original PLATO were also significant in terms of DoE evaluated at the highest reached percentile. The non-statistically significant finding of more bleeding events in the ticagrelor group in the original PLATO study (HR 1.04, $95 \%$ CI 0.95 to 1.13 ) corresponded to a non-statistically significant negative DoE of -29 days (95\% CI 88 to 30 ) at the 10th percentile (panel $\mathrm{E}$ of the figure). Findings from the current study may add substantial information to the pharmaco-pathophysiological understanding of how the treatment provides its effect and how that relates to time on treatment. In general, novel approaches, such as the evaluation of survival percentiles, should always be complemented with the presentation of results from classical approaches to provide a more comprehensive understanding of the treatment effect.

\section{CONCLUSION}

This study confirmed evidence of beneficial effects of ticagrelor over clopidogrel, and provided the magnitude of such effects in terms of delayed event time. Through this practical example in an important clinical trial, we presented the advantages that the evaluation of additional measures of effect may accrue to the literature. In 


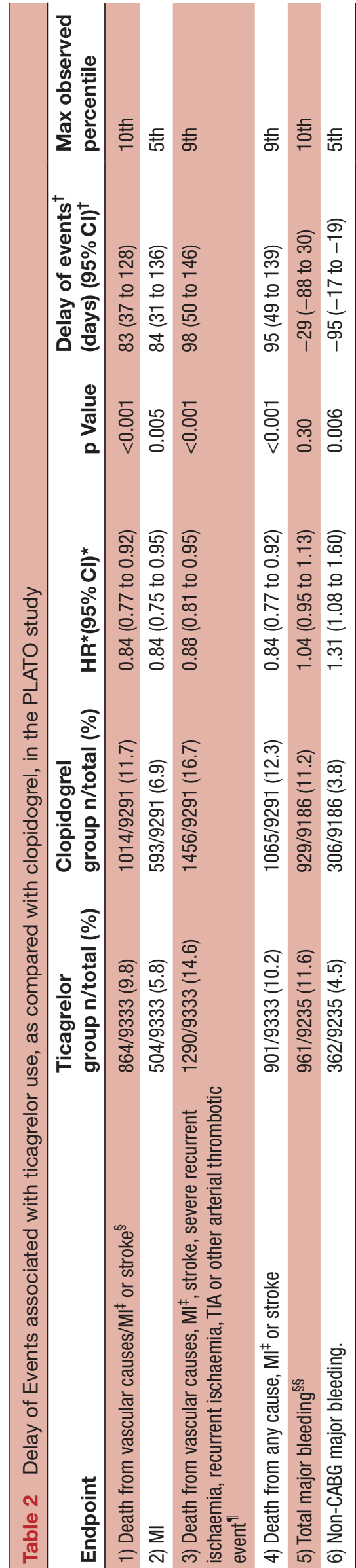

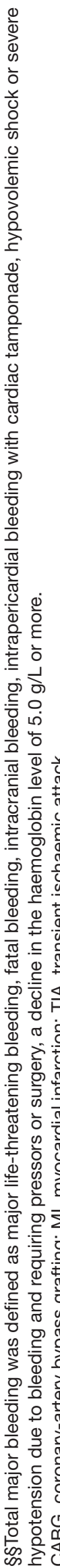

particular, investigating time-to-event data with a percentile approach allows presenting treatment effects from randomised controlled studies as absolute measures of the time by which an event may be delayed due to the treatment.

Acknowledgements Publications management was provided by Ebba Bergman, $\mathrm{PhD}$, at Uppsala Clinical Research Centre, Uppsala, Sweden.

Contributors LW, SKJ, CPC and AH designed the PLATO trial. AB, NO, JS and PL developed the aims and the analysis of this study. HR performed statistical analysis and produced tables and figures. $A B$ and PL drafted the manuscript. All authors critically interpreted the results and reviewed the manuscript, giving their final consent to the final version.

Funding The PLATO study was funded by AstraZeneca. Support for the analysis and interpretation of results and preparation of the manuscript was provided through funds to the Uppsala Clinical Research Center and Duke Clinical Research Institute as part of the Clinical Study Agreement.

Competing interests ABE: None. L Wallentin: institutional research grants, consultancy fees, lecture fees, and travel support from Bristol-Myers Squibb/ Pfizer, AstraZeneca, GlaxoSmithKline, Boehringer Ingelheim; institutional research grants from Merck \& Co, Roche; consultancy fees from Abbott; holds two patents involving GDF-15. NO: None. S James: institutional research grant, honoraria and consultant/advisory board fee fromAstraZeneca; institutional research grant and consultant/advisory board fee from Medtronic; institutional research grants and honoraria from The Medicines Company; consultant/advisory board fees from Janssen, Bayer. CPC: research grants from AstraZeneca, Takeda, GlaxoSmithKline, Boerhingerlngelheim, Merck, Arisaph, Janssen, Accumetrics; consultant fees from GlaxoSmithKline, Takeda, Merck, Bristol-Myers Squibb, Alnylam, Pfizer, Essentialis, Kowa, Lipimedix, Regeneron, Sanofi, Boerhinger Ingelheim; travel support from AstraZeneca, Boerhingerlngelheim; personal fees from CSL Behring. A H: employee of AstraZeneca. JS: None. H Renlund: institutional research grant from AstraZeneca. PL: None.

Provenance and peer review Not commissioned; internally peer reviewed

Data sharing statement Data from this study are unavailable. Further information can be obtained by contacting the PLATO committe at Uppsala University." This information can be included where most appropriate.

Open Access This is an Open Access article distributed in accordance with the Creative Commons Attribution Non Commercial (CC BY-NC 4.0) license, which permits others to distribute, remix, adapt, build upon this work non-commercially, and license their derivative works on different terms, provided the original work is properly cited and the use is non-commercial. See: http://creativecommons.org/ licenses/by-nc/4.0/

(C) Article author(s) (or their employer(s) unless otherwise stated in the text of the article) 2017. All rights reserved. No commercial use is permitted unless otherwise expressly granted.

\section{REFERENCES}

1. Wallentin L, Becker RC, Budaj A, et al. Ticagrelor versus clopidogrel in patients with acute coronary syndromes. N Engl J Med 2009;361:1045-57.

2. Anderson JL, Adams CD, Antman EM, et al. 2012 ACCF/AHA focused update incorporated into the ACCF/AHA 2007 guidelines for the management of patients with unstable angina/non-ST-elevation myocardial infarction: a report of the American College of Cardiology Foundation/American Heart Association Task Force on Practice guidelines. J Am Coll Cardiol 2013;61:e179-347.

3. Steg PG, James SK, Gersh BJ. 2012 ESC STEMI guidelines and reperfusion therapy: evidence-based recommendations, ensuring optimal patient management. Heart 2013;99:1156-7.

4. Roffi M, Patrono C, Collet JP, et al. 2015 ESC guidelines for the management of acute coronary syndromes in patients presenting without persistent ST-segment elevation: task Force for the management of acute coronary syndromes in patients presenting without persistent ST-Segment elevation of the European Society of Cardiology (ESC). Eur Heart J 2016;37:267-315.

5. Moher D, Hopewell S, Schulz KF, et al. CONSORT 2010 explanation and elaboration: updated guidelines for reporting parallel group randomised trials. BMJ 2010;340:c869.

6. Hernán MA. The hazards of hazard ratios. Epidemiology 2010;21:13-15. 
7. Uno $\mathrm{H}$, Wittes J, Fu H, et al. Alternatives to Hazard Ratios for comparing the efficacy or safety of therapies in noninferiority sStudies. Ann Intern Med 2015;163:127-34.

8. Uno $\mathrm{H}$, Claggett $\mathrm{B}$, Tian $\mathrm{L}$, et al. Moving beyond the hazard ratio in quantifying the between-group difference in survival analysis. $J$ Clin Oncol 2014;32:2380-5.

9. Orsini N, Wolk A, Bottai M. Evaluating percentiles of survival. Epidemiology 2012;23:770-1.

10. Lytsy P, Berglund L, Sundström J. A proposal for an additional clinical trial outcome measure assessing preventive effect as delay of events. Eur J Epidemiol 2012;27:903-9.

11. Bottai M, Zhang J. Laplace regression with censored data. Biom J 2010;52:487-503.

12. Bellavia ABM, Orsini N. Evaluating additive interaction using survival percentiles. Epidemiology. In Press. 2015.

13. Berglund E, Westerling R, Sundström J, et al. Treatment effect expressed as the novel delay of event measure is associated with high willingness to initiate preventive treatment-A randomized survey experiment comparing effect measures. Patient Educ Couns 2016;99:2005-11.

14. Dahl R, Gyrd-Hansen D, Kristiansen IS, et al. Can postponement of an adverse outcome be used to present risk reductions to a lay audience? A population survey. BMC Med Inform Decis Mak 2007;7:8.

15. James S, Akerblom A, Cannon CP, et al. Comparison of ticagrelor the first reversible oral P2Y(12) receptor antagonist, with clopidogrel in patients with acute coronary syndromes: rationale, design, and baseline characteristics of the PLATelet inhibition and patient outcomes (PLATO) trial. Am Heart J 2009;157:599-605.
16. Frumento $\mathrm{P}$, Bottai $\mathrm{M}$. An estimating equation for censored and truncated quantile regression. Comput Stat Data Anal 2016 http://dx. doi.org.proxy.kib.ki.se/

17. Bobbio M, Demichelis B, Giustetto G. Completeness of reporting trial results: effect on physicians' willingness to prescribe. Lancet 1994;343:1209-11.

18. Naylor CD, Chen E, Strauss B. Measured enthusiasm: does the method of reporting trial results alter perceptions of therapeutic effectiveness? Ann Intern Med 1992;117:916-21.

19. Hux JE, Naylor CD. Communicating the benefits of chronic preventive therapy: does the format of efficacy data determine patients' acceptance of treatment? Med Decis Making 1995;15:152-7

20. Bellavia A, Discacciati A, Bottai M, et al. Using laplace regression to model and predict percentiles of age at death when age is the primary time scale. Am J Epidemiol 2015;182:271-7.

21. Bottai M, Orsini N, Geraci M. A gradient search maximization algorithm for the Asymmetric Laplace likelihood. Journal of Statistical Computation and Simulation 2014:1-7.

22. Bottai M, Orsini N. A command for Laplace regression. Stata J 2013;13:302-14.

23. Bellavia A, Larsson SC, Bottai M, et al. Fruit and vegetable consumption and all-cause mortality: a dose-response analysis. Am J Clin Nutr 2013;98:454-9.

24. Rizzuto D, Orsini N, Qiu C, et al. Lifestyle, social factors, and survival after age 75: population based study. BMJ 2012;345:e5568.

25. Bellavia A, Åkerstedt T, Bottai M, et al. Sleep duration and survival percentiles across categories of physical activity. Am J Epidemiol 2014;179:484-91. 